\title{
Thiol-disulfide status of human sperm proteins
}

\author{
J. Seligman ${ }^{1}$, N. S. Kosower ${ }^{1}$, R. Weissenberg ${ }^{2}$ and R. Shalgi ${ }^{3 *}$ \\ Departments of ${ }^{1}$ Human Genetics and ${ }^{3}$ Embryology, Sackler School of Medicine, Tel Aviv University, \\ Israel; and ${ }^{2}$ Institute of Endocrinology, Sheba Medical Center, Tel Hashomer, Israel
}

\begin{abstract}
The thiol-disulfide status in proteins of human spermatozoa categorized as normozoospermic, teratozoospermic and asthenozoospermic was examined. Washed spermatozoa were incubated with or without dithiothreitol (DTT) to reduce disulfides (SS) to thiols (SH), and then labelled with the specific fluorescence thiol labelling agent monobromobimane $(\mathrm{mBBr})$. The $\mathrm{SH}$ and $\mathrm{SS}$ in intact labelled spermatozoa were evaluated by fluorescence microscopy and by flow cytometry analysis; mBBr-labelled spermatozoa were solubilized and sperm proteins analysed by gel electrophoresis (SDS-PAGE for non-basic, whole sperm proteins and acid urea-PAGE for sperm nuclear basic proteins). Microscopy and flow cytometry showed that normozoospermic samples (having normal sperm count, morphology and motility) contained both SH and SS, with more SS than SH. Heterogeneity in the proportion of $\mathrm{SH} /(\mathrm{SH}$ plus $\mathrm{SS}$ ) was observed among spermatozoa within the ejaculates. The total SH plus SS was similar among the ejaculates, with some variability in $\mathrm{SH} / \mathrm{SH}$ plus SS) noted among them. SDS-PAGE of solubilized normozoospermic cells showed differences in the $\mathrm{SH}$ and SS content of the protein bands. Acid urea-PAGE of basic proteins isolated from normozoospermic samples showed protamines $\mathrm{P} 1$ and $\mathrm{P} 2$ and traces of non-protamine basic proteins. $\mathrm{P} 1$ and $\mathrm{P} 2$ contained $\mathrm{SH}$ and SS, with variability in $\mathrm{SH} /(\mathrm{SH}$ plus SS) observed among the samples. Teratozoospermic samples (in which $>90 \%$ of the spermatozoa exhibited abnormal morphology) were similar in thiol-disulfide status to normozoospermic samples, but contained non-protamine basic proteins in addition to protamines. Spermatozoa in asthenozoospermic samples (in which $>90 \%$ of the spermatozoa were immotile) contained lower amounts of $\mathrm{SH}$ than did those of normozoospermic samples; the total (SH plus SS) in the asthenozoospermic samples was similar to that in the normozoospermic samples, as shown by microscopy, flow cytometry and gel electrophoresis. The significantly lower $\mathrm{SH} /(\mathrm{SH}$ plus $\mathrm{SS}$ ) was evident in most sperm proteins, including the protamines, of the asthenozoospermic samples. This 'over oxidation' of sperm thiols may result from an abnormal maturation in the epididymis or from an effect of the seminal plasma.
\end{abstract}

\section{Introduction}

During the final stages of mammalian spermatogenesis, the histones associated with the DNA are replaced by protamines, low molecular mass basic proteins containing a large number of cysteine and arginine residues. The DNA-protamine complex acquires a highly compact, inert and transcriptionally inactive form (Gatewood et al., 1987). Further stabilization of sperm nuclei occurs during the passage of the spermatozoa through the epididymis. It was proposed that oxidation of the protamine cysteine residues to disulfides plays a role in stabilization of the sperm nucleus in various species, including humans (Calvin and Bedford., 1971; Marushige and Marushige, 1975; Miller and Masui, 1982; Pellicciari et al., 1983; Seligman and Shalgi, 1991). During sperm maturation, tail structures are also stabilized, mainly through oxidation of thiol groups (Bedford

\footnotetext{
*Correspondence.
}

Revised manuscript received 13 December 1993. and Calvin, 1974; Shalgi et al., 1989). It was proposed that sperm thiol oxidation is important for sperm motility (Cornwall et al., 1988), for sperm capacitation and acrosome reaction (Fleming et al., 1986; Yanagimachi et al., 1983), for protecting chromatin from autolysis in the female genital tract (Marushige and Marushige, 1975) and for sperm interaction with the egg membrane (Yanagimachi et al., 1983).

We studied the thiol-disulfide status of rat spermatozoa using the fluorescent thiol labelling agent monobromobimane $(\mathrm{mBBr})$ (Kosower and Kosower, 1987) and showed that during sperm epididymal maturation thiol oxidation occurs in protamine and in many sperm tail protein fractions (Shalgi et al., 1989; Seligman and Shalgi, 1991). In contrast to the rat, in which most of the mature spermatozoa within and among samples appear to be similar with respect to sperm thiol and disulfide content, human spermatozoa displayed a significant heterogeneity in thiol content among spermatozoa having similar morphology, as observed by microscopy and by flow cytometry of mBBr-labelled spermatozoa (Seligman et al., 1991; 
Rufas et al., 1991). The rapid epididymal transport of spermatozoa in man (Amann and Howards, 1980) may play a role in the appearance of immature spermatozoa in normal semen and the heterogeneity observed in the sperm thiol content. In addition, the difference between rat and human spermatozoa may be due in part to differences in chromatin organization. The rat sperm chromatin contains protamine 1 , whereas the human spermatozoa contains protamines 1 and 2 . It has been proposed that the presence of protamine 2 in spermatozoa facilitates nuclear decondensation (Perreault et al, 1988).

In the present work we used $\mathrm{mBBr}$ to evaluate the protein thiol-disulfide status in human spermatozoa in semen samples categorized as normozoospermic, teratozoospermic and asthenozoospermic according to routine semen analysis parameters. Thiols and disulfides in intact, mBBr-labelled spermatozoa were evaluated by fluorescence microscopy and by flow cytometry. Whole sperm proteins and basic proteins were prepared from each sperm sample and analysed by gel electrophoresis. The results indicate an altered thiol-disulfide status ('over oxidation') in the asthenozoospermic samples.

\section{Materials and Methods}

\section{Sperm samples}

Men who were referred to the male fertility clinic at Sheba Medical Center as male partners of infertile couples were included in this study. Sperm samples were obtained by masturbation, after 2-3 days of sexual abstinence. Samples were allowed to liquefy at room temperature for $30 \mathrm{~min}$. Semen volume was measured and sperm concentration and motility were assessed. Sperm morphology was evaluated following fixation and Papanicolaou staining. Sperm samples were categorized according to the criteria defined by the World Health Organization (1980), as normozoospermic, oligospermic, teratospermic or asthenozoospermic. Semen parameter criteria for the normozoospermic samples were: sperm count $>30 \times 10^{6}$ spermatozoa $\mathrm{ml}^{-1}$, volume of $2-5 \mathrm{ml}$, normal morphology of $>40 \%$ and motility $>40 \%$ of the spermatozoa. A sample was categorized as abnormal when it showed a subnormal level of one parameter and the other parameters were in the normal range. Oligozoospermic samples (sperm count $<20 \times 10^{6} \mathrm{ml}^{-1}$ ) were not included in this work because their low sperm count did not allow a reliable protein thiol analysis by the available methods. The samples studied included 34 normozoospermic samples, 16 teratozoospermic samples (with abnormal morphology in $>90 \%$ of the spermatozoa) and 10 asthenozoospermic samples (with lack of motility in $>90 \%$ of the spermatozoa). Azoospermic samples, which do not contain spermatozoa, were also examined and served as a baseline for sperm protein analysis by electrophoresis.

\section{Labelling of spermatozoa with $\mathrm{mBBr}$}

Seminal fluid was removed by washing samples twice by suspension in buffer containing $10 \mathrm{mmol}$ Tris- $\mathrm{HCl} \mathrm{l}^{-1}$, $140 \mathrm{mmol} \mathrm{NaCl} \mathrm{l}{ }^{-1}, \mathrm{pH} 7.4$ (TBS) and centrifuging at $600 \mathrm{~g}$ for $10 \mathrm{~min}$. Spermatozoa were resuspended in TBS to $4-10 \times 10^{6}$ cells $\mathrm{ml}^{-1}$ and aliquots incubated at $37^{\circ} \mathrm{C}$ for
$10 \mathrm{~min}$ with or without $1.0 \mathrm{mmol}$ dithiothreitol $\mathrm{1}^{-1}$ (DTT), to reduce sperm disulfides to reactive thiols. Samples were then washed twice, resuspended in TBS and labelled with $\mathrm{mBBr}$, as described by Shalgi et al. (1989) (mBBr was obtained from E. M. Kosower, School of Chemistry, Tel Aviv University). A stock solution of $50 \mathrm{mmol} \mathrm{mBBr} \mathrm{l}^{-1}$ in acetonitrile (stable for months when kept in the dark) was added to sperm sus-

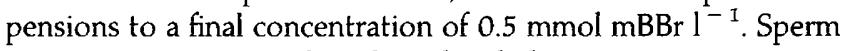
suspensions were incubated in the dark at room temperature for $10 \mathrm{~min}$, and then washed twice and resuspended in TBS. In some experiments, aliquots of spermatozoa, kept in seminal fluid or washed and resuspended in TBS, were incubated at $4^{\circ} \mathrm{C}$ for 3 days, and then washed and labelled with $\mathrm{mBBr}$.

\section{Isolation of sperm nuclear basic proteins}

Sperm nuclear proteins were isolated as described by Gusse et al. (1986) with some modifications. $\mathrm{mBBr}$-labelled sperm suspensions were incubated with $10 \mathrm{mmol}$ DTT $\mathrm{l}^{-1}$ for $20 \mathrm{~min}$ at $37^{\circ} \mathrm{C}$ in the presence of $1 \mathrm{mmol}$ EDTA $\mathrm{l}^{-1}$ and $0.2 \mathrm{mmol}$ phenylmethylsulfonyl fluoride $1^{-1}$ (PMSF). $N$-Ethylmaleimide (NEM) was then added to a final concentration of $25 \mathrm{mmol} \mathrm{l}^{-1}$ and incubation continued for a further $40 \mathrm{~min}$, followed by a short sonication (to separate heads from tails). For chromatin isolation, spermatozoa were incubated for $30 \mathrm{~min}$ at room temperature with $1 \%$ mixed alkyltrimethylammonium bromide to solubilize sperm tail, acrosome and nuclear membrane. Sperm nuclei were washed and proteins were extracted overnight at $4^{\circ} \mathrm{C}$ by $0.25 \mathrm{~mol} \mathrm{HCl} 1^{-1}$. $\mathrm{HCl}$-soluble proteins were precipitated by $20 \%$ trichloroacetic acid (TCA), recovered by centrifugation, and washed with acidified acetone and acetone.

\section{Evaluation of sperm thiols by microscopy}

The $\mathrm{mBBr}$-labelled spermatozoa were examined using a Leitz microscope equipped with epifluorescence ultraviolet (UV) illumination and filter $A$ (for excitation range of $340-380 \mathrm{~nm}$, and emission above $450 \mathrm{~nm}$ ). Samples were photographed twice, using transmitted light and then UV illumination. Photography was carried out with Kodak TMax film 400 ASA and identical exposure times.

\section{Analysis of sperm thiols by flow cytometry}

Flow cytometry of mBBr-labelled spermatozoa was performed using a FACS IV flow cytometer (Beckton Dickinson Corp., Mountain View, CA) equipped with an argon-ion laser (Coherent Model Innova 90-5) tuned to UV (340-360 nm) and operated at $100 \mathrm{~mW}$ output, in the light stabilized mode. Glutaraldehyde-fixed chicken erythrocytes were used to standardize the instrument. The fluorescence of $10^{4}$ spermatozoa in each sample was measured with a $400 \mathrm{~nm}$ LP filter for light emission. Data were processed using the Consort 40 Beckton Dickinson Corp. analysis programs.

\section{Sperm protein analysis by gel electrophoresis}

An aliquot of mBBr-labelled spermatozoa was solubilized in $5 \%$ SDS and $3 \%$ mercaptoethanol. Solubilized sperm samples 
Table 1. Evaluation of human sperm thiol status by fluorescence microscopy

\begin{tabular}{lcc}
\hline Samples & $\begin{array}{c}\text { Fluorescence in } \\
\text { sperm head }\end{array}$ & $\begin{array}{c}\text { Fluorescence in } \\
\text { sperm tail }\end{array}$ \\
\hline $\begin{array}{l}\text { Normozoospermic } \\
\text { Asthenozoospermic }\end{array}$ & $\begin{array}{c}20-50(+ \text { to }+++)^{\mathrm{b}} \\
5-10(+ \text { to }++)^{\mathrm{b}}\end{array}$ & $\begin{array}{c}>70 \quad(+)^{\mathrm{b}} \\
5-10(+)^{\mathrm{b}}\end{array}$
\end{tabular}

apercentage of spermatozoa exhibiting fluorescence.

${ }^{\mathrm{b}}$ The degree of fluorescence was estimated as weak $(+)$, moderate $(++)$ or strong $(+++)$.

were electrophoresed on SDS-PAGE electrophoresis as described by Laemmli (1970). Nuclear basic proteins were solubilized in $5 \%$ acetic acid, $3 \%$ mercaptoethanol, $8 \mathrm{~mol}$ urea $1^{-1}$ and $15 \%$ sucrose and electrophoresis was performed on acid-urea PAGE as described by Panyim and Chalkley (1969). After electrophoretic separation, gels were photographed using UV illumination to detect the fluorescent protein bands. Gels were then stained with Coomassie blue and photographed again. Densitometric profiles of fluorescent and stained gels were performed on the films of the gels. The densitometry peaks of the proteins (Coomassie blue stained profiles) were normalized to the same value, so that the fluorescence intensities of the different samples could be compared.

\section{Results}

\section{Evaluation of sperm thiols and disulfides by microscopy}

Spermatozoa were labelled with $\mathrm{mBBr}$ and examined under a fluorescence microscope. Samples categorized as normozoospermic showed variability in fluorescence among cells with normal morphology, especially in the heads. While some spermatozoa displayed high fluorescence in the heads, others were completely dark (Fig. 1a,c). The fluorescence intensity of teratozoospermic samples was similar to that of spermatozoa in normozoospermic samples. Asthenozoospermic samples had a significantly lower percentage of fluorescent spermatozoa than did the normozoospermic samples, and most cells showed little or no fluorescence (Fig. Ib,d). The percentages of sperm heads and tails in the normozoospermic and asthenozoospermic samples that exhibited fluorescence and the degree of fluorescence intensity, as evaluated by fluorescence microscopy are summarized in Table 1. After DTT pretreatment, mBBr-labelled spermatozoa from all samples exhibited a high fluorescence intensity, especially in the heads, and most cells appeared similar (not shown).

\section{Analysis of sperm thiols and disulfides by flow cytometry}

Spermatozoa were incubated with or without DTT, and then labelled with $\mathrm{mBBr}$. Flow cytometry analysis of spermatozoa from normozoospermic samples showed that the DTTpretreated spermatozoa exhibited significantly more fluorescence and appeared more homogeneous than did the samples incubated without DTT (Fig. 2a). Flow cytometry histograms
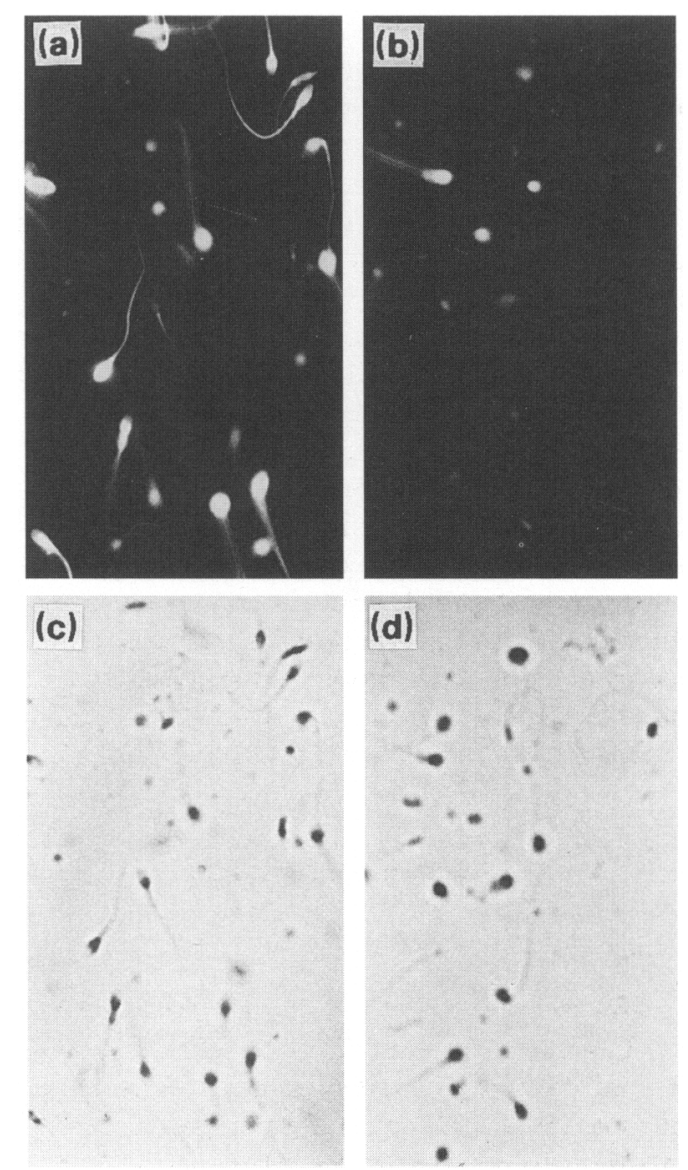

Fig. 1. Micrographs of human spermatozoa labelled with monobromobimane $(\mathrm{mBBr})$. (a,b) Fluorescence micrographs and (c,d) photomicrographs. $(a, c)$ A normozoospermic sample and $(b, d)$ an asthenozoospermic sample.

of teratozoospermic samples appeared similar to those of normozoospermic samples (not shown). Flow cytometry histograms of asthenozoospermic samples incubated without DTT were shifted to the left compared with those of normozoospermic samples (Fig. 2a,b), indicating a lower content of thiols in the asthenozoospermic cells than in the normozoospermic cells (Fig. 2b). After pretreatment with DTT, the histograms of these samples were similar to those of the normozoospermic samples, indicating that the total amount of sperm thiols and disulfides (SH plus SS) was similar in these groups. The proportion of reactive thiols (i.e., $\mathrm{SH} /(\mathrm{SH}$ plus $\mathrm{SS}$ )) was estimated from the mean fluorescence of histograms of spermatozoa incubated without DTT and the fluorescence of DTT-pretreated cells. The percentage of reactive $\mathrm{SH}$ was (mean \pm SEM) $16.6 \pm 1.3 \%$ $(n=9)$ and $9.7 \pm 1.24 \%(n=9)$ in the normozoospermic and asthenozoospermic samples, respectively $(P<0.01)$.

\section{Sperm protein analysis by gel electrophoresis}

Proteins of solubilized spermatozoa were analysed by SDSPAGE. When stained by Coomassie blue, all normozoospermic samples displayed a series of protein bands ranging in molecular mass from about $90 \mathrm{kDa}$ to $14 \mathrm{kDa}$ with one major, wide 


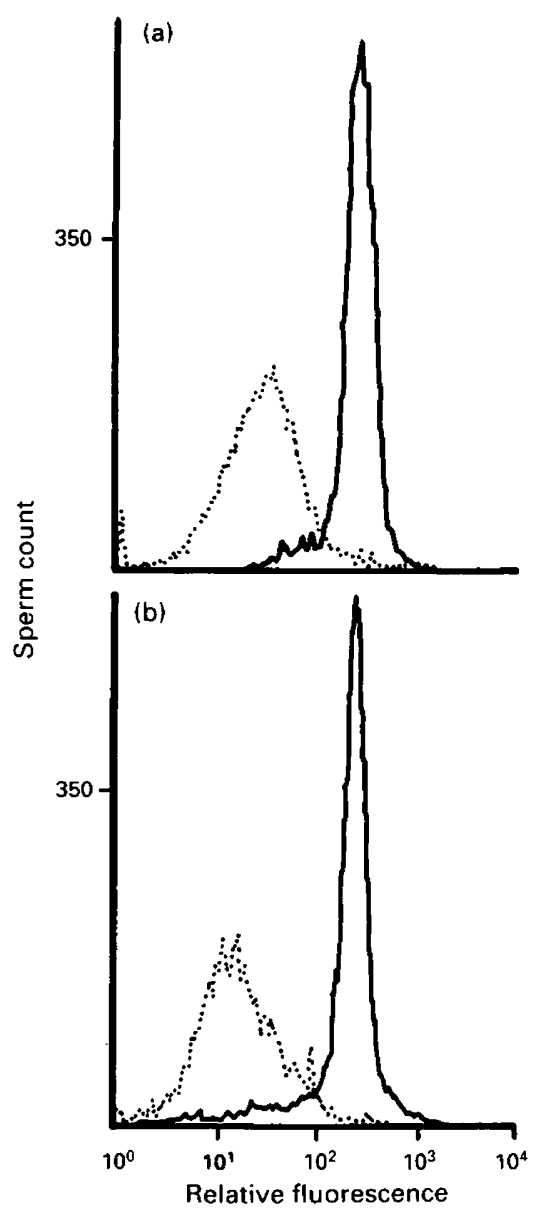

Fig. 2. Flow cytometry histograms of untreated $(\cdots)$ and DTT-treated $(\stackrel{)}{\longrightarrow}$ human spermatozoa labelled with monobromobimane $(\mathrm{mBBr})$. (a) A normozoospermic sample and (b) an asthenozoospermic sample.

protein band at about $16 \mathrm{kDa}$ (Fig. 3a, lanes 1, 2). Overall sperm protein profiles of the various normozoospermic samples were similar, although there were slight differences in the relative amounts of some protein bands among the different samples. Azoospermic samples (semen samples devoid of spermatozoa) showed only traces of several bands (not shown), indicating that the proteins of the samples analysed by PAGE were sperm proteins, with negligible contamination by other cells or semen fluid proteins.

The thiol-disulfide status in proteins obtained from normozoospermic samples were characterized by comparing fluorescence and Coomassie blue stained protein profiles. Spermatozoa from the normozoospermic samples presented some fluorescent protein bands, including some high molecular mass bands and a low molecular mass wide protein band of about 14-16 kDa (Fig. 3a, lane 3). Pretreatment of spermatozoa with DTT before labelling with $\mathrm{mBBr}$ resulted in a significant increase in fluorescence of the bands, indicating the presence of appreciable amounts of protein disulfides in these spermatozoa (Fig. 3a, lanes 3, 4).

The fluorescence of the protein bands as compared to the protein content is illustrated by densitometry of the fluorescent and the Coomassie blue stained profiles (Fig. 3b). In the absence (a)

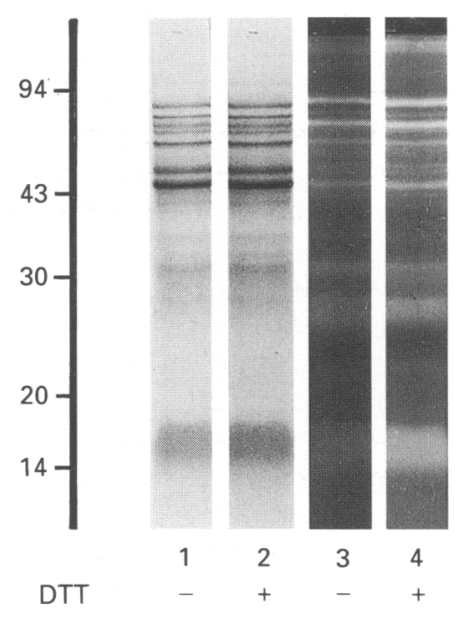

(b)
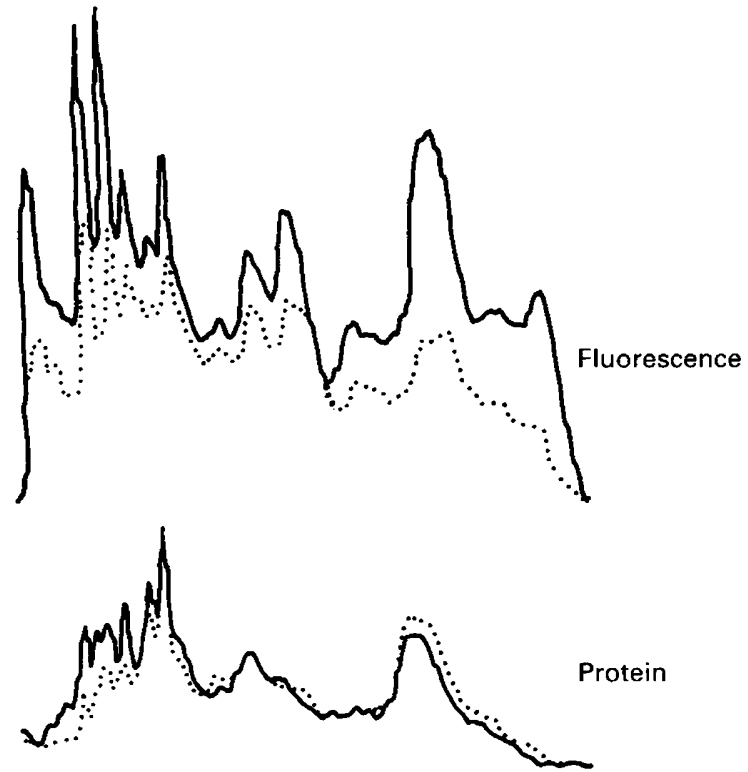

Fig. 3. (a) SDS-PAGE of human sperm proteins from normozoospermic samples labelled with monobromobimane $(\mathrm{mBBr})$. Lanes 1 and 2: Coomassie blue stain; lanes 3 and 4: fluorescence illumination. Lanes 1 and 3 untreated and lanes 2 and 4 DTT treated. Approximate molecular masses are as indicated from migration of known protein standards $\left(\times 10^{-3}\right)$. (b) Densitometry of the fluorescence and Coomassie blue stained profiles. Untreated ( $\cdots$ ) (see lanes 1, 3); DTT treated $(\longrightarrow$ ) (see lanes 2,4$)$. The densitometry peaks of the proteins (Coomassie blue stained profiles) were normalized to the same value, so that the fluorescence intensities of the untreated and DTT-treated samples could be compared.

of DTT, little fluorescence was evident in most protein bands. Two protein bands (between 80 and $90 \mathrm{kDa}$ ) stained weakly in Coomassie blue and displayed relatively high fluorescence, while other bands presented little fluorescence relative to protein content. An increase in the fluorescence of most bands was observed after DTT pretreatment of the spermatozoa. A high molecular mass fraction at the origin of the gel and the 
protein band of about $14-16 \mathrm{kDa}$ exhibit a significant increase in fluorescence following DTT pretreatment. The fluorescence profiles of most normozoospermic samples were similar, with only slight variations observed among them. In several of the normozoospermic samples a somewhat higher protein fluorescence was noted. Fluorescence and stained protein profiles of teratozoospermic samples were similar to those of the normozoospermic samples.

Asthenozoospermic samples showed stained protein profiles similar to those of normozoospermic samples, with slight variations in the relative amounts of some protein bands (Fig. $4 a$, lanes 1,2 compared with Fig. $3 a$, lanes 1,2 ). However, the fluorescence of asthenozoospermic proteins was significantly lower than that of the normozoospermic proteins, and little or no fluorescence was observed in most protein bands relative to the amount of the protein in the bands (Fig. 4a, lanes 1, 3). The difference between the asthenozoospermic and normozoospermic proteins is especially evident in the two protein bands of $80-90 \mathrm{kDa}$, which in the normozoospermic samples showed a significant fluorescence. DTT pretreatment caused a significant increase in the fluorescence of all protein bands, and fluorescence intensity became similar to that of DTT-pretreated normozoospermic samples (Fig. 4a, lanes 3, 4 compared with Fig. 3a, lanes 3,4). Densitometry of the fluorescence and of Coomassie blue stained profiles (Fig. 4 b) demonstrated the lack of or very low thiol content in most protein bands and the very significant increase in the fluorescence of these proteins after DTT pretreatment of the spermatozoa.

Electrophoresis of the nuclear basic proteins extracted from mBBr-labelled spermatozoa of normozoospermic samples displayed two major protamine bands. In some samples, one or two additional minor basic protein fractions were observed, as shown in the Coomassie blue stained profiles (Fig. 5, lanes I-3). Fluorescence was observed in the protamine bands of all normozoospermic samples, with somewhat more fluorescence noted in the slower moving protamine, protamine $1(\mathrm{P} 1)$ than in protamine $2(\mathrm{P} 2)$ relative to the protein content (Fig. 5, densitometry profiles). Variability in the fluorescence intensity was noted among different normozoospermic samples (Fig. 5, lanes 1, 2, 3). Protamines extracted from one sperm sample categorized as normozoospermic exhibited high fluorescence (Fig. 5, lane 1). This sample showed especially high fluorescence also in proteins analysed by SDS-PAGE. After DTT treatment, all the samples exhibited a similar protamine fluorescence (not shown).

Electrophoresis of basic proteins extracted from spermatozoa of teratozoospermic patients (Fig. 5a, lanes 4,5) showed the two major protamine bands and the additional basic protein band(s) which seemed to be more prominent than in the normozoospermic samples. Some variability in protamine fluorescence was noted among the teratozoospermic samples (Fig. 5, lanes 4, 5). Asthenozoospermic samples also presented two bands of protamines. A lower ratio of P1:P2 was observed in several samples, compared with that in normozoospermic samples (Fig. 6a, lanes 1, 2 compared to Fig. 6b, lanes 1, 2). Protamines extracted from asthenozoospermic samples showed very little fluorescence compared with the protamines in normozoospermic samples that exhibited some fluorescence (Fig. 6a, lane 1 compared to Fig. 6b, lane 1). Protamines extracted from DTT-pretreated, mBBr-labelled asthenozoo- (a)

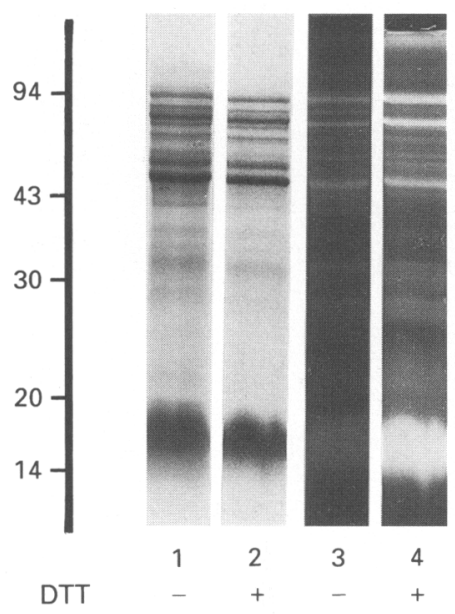

(b)

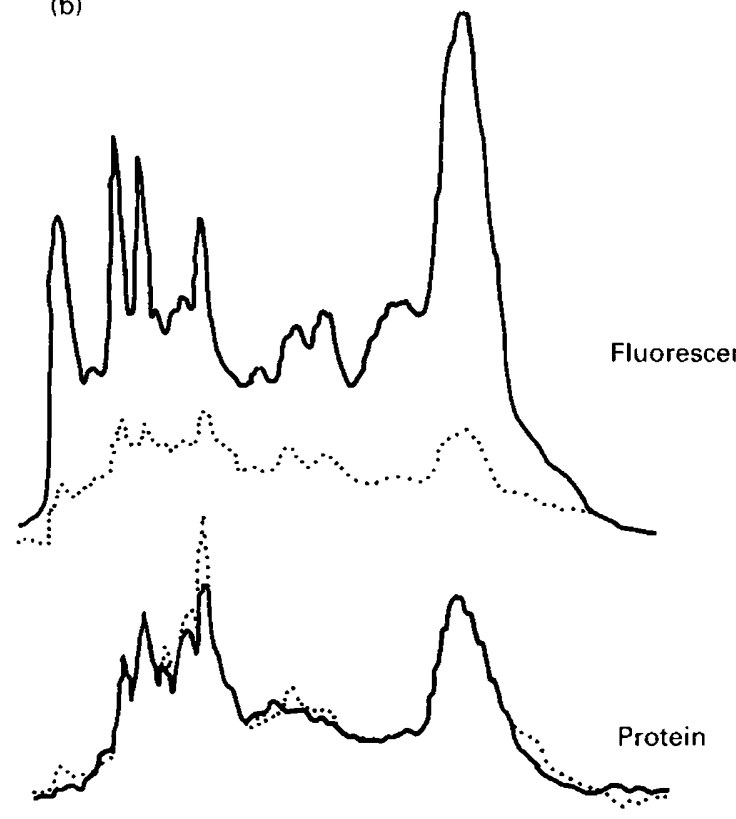

Fig. 4. (a) SDS-PAGE of human sperm proteins from asthenozoospermic samples labelled with monobromobimane $(\mathrm{mBBr})$. Lanes 1 and 2 : Coomassie blue stain; lanes 3 and 4 fluorescence illumination. Lanes I and 3 untreated and lanes 2 and 4 DTT treated. Approximate molecular masses are as indicated from migration of known protein standards $\left(\times 10^{-3}\right)$. (b) Densitometry of the fluorescence and Coomassie blue stained profiles. Untreated $(\cdots)$ and DTT-treated $(-)$. The densitometry peaks of the proteins (Coomassie blue stained profiles) were normalized to the same value, so that fluorescence intensities of the untreated and DTT treated samples could be compared.

spermic samples exhibited a high level of fluorescence (Fig. $6 \mathrm{~b}$, lane 2) similar to that of protamines extracted from DTTpretreated normozoospermic samples (Fig. 6a, lane 2). The differences in the fluorescence behaviour between the protamine bands in normozoospermic and in asthenozoospermic samples are demonstrated by densitometry of the fluorescent profiles (Fig. 6). 
(a)
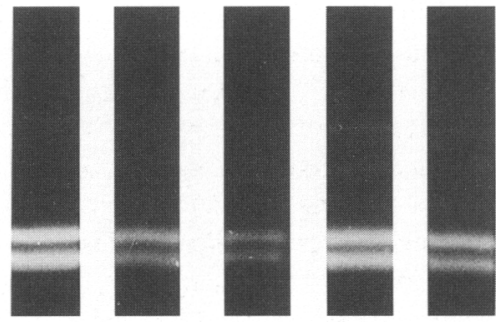

(b)

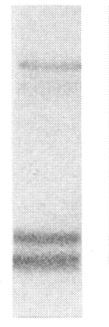

1

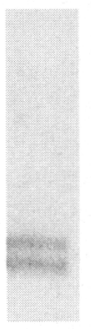

2

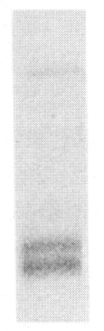

3
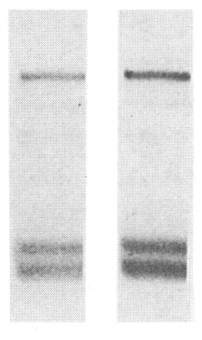

5

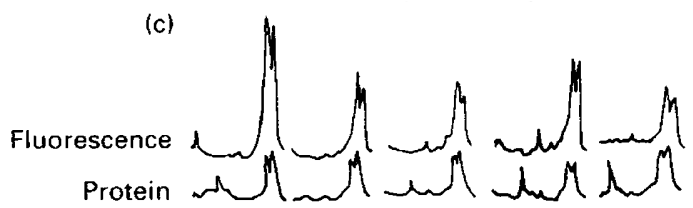

Fig. 5. Acid urea-PAGE of basic proteins extracted from nuclei of monobromobimane ( $\mathrm{mBBr}$ )-labelled human spermatozoa. Lanes 1-3: three normozoospermic samples; lanes 4 and 5 two teratozoospermic samples. (a) Fluorescence illumination. (b) Coomassie blue stain. (c) Densitometry of the fluorescence and protein profiles (see lanes 1-5). The densitometry peaks of the proteins (Coomassie blue profiles) were normalized to the same value, so that the fluorescence intensities of the different samples could be compared.

\section{The effects of seminal fluid on human sperm thiols}

Aliquots of spermatozoa, washed and resuspended in TBS or kept in the seminal fluid, were incubated at $4^{\circ} \mathrm{C}$ for 3 days, and then labelled with $\mathrm{mBBr}$ and analysed by flow cytometry. The histogram of washed spermatozoa incubated in TBS for 3 days was similar to that of fresh, non-incubated spermatozoa (Fig. $7 \mathrm{a}, \mathrm{b})$. Spermatozoa that were incubated in seminal fluid had a lower thiol content, as shown by a shift of the histogram to the left (Fig. 7c). After DTT pretreatment, all samples showed similar histograms (not shown), indicating that the lower thiol content in the spermatozoa kept in the seminal fluid was due to the oxidation of thiols to disulfides in these spermatozoa. The possibility that thiol oxidation occurred because of bacterial or fungal contamination was excluded by incubating spermatozoa in the presence or absence of azide. Incubation with azide had no effect on the flow cytometry histograms of $\mathrm{mBBr}$-labelled spermatozoa.

\section{Discussion}

Oxidation of thiols is an integral part of mammalian sperm maturation during epididymal passage (Calvin and Bedford, 1971; Marushige and Marushige, 1975; Pellicciari et al., 1983; Huang et al., 1984; Shalgi et al., 1989). The epididymal transit (a)

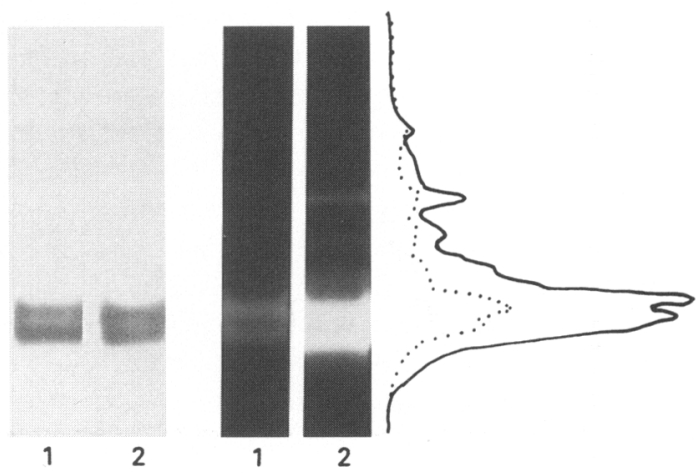

(b)

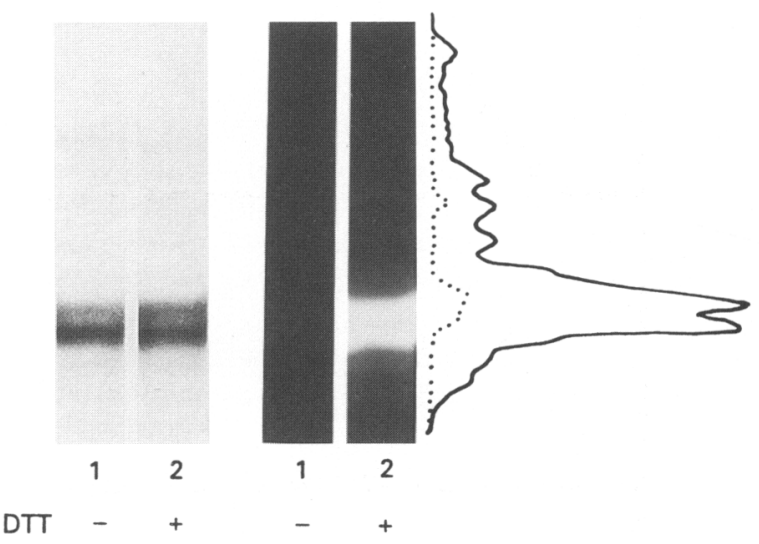

Fig. 6. Acid urea-PAGE of basic proteins extracted from monobromobimane ( $\mathrm{mBBr}$ )-labelled human spermatozoa. (a) A normozoospermic sample; (b) an asthenozoospermic sample. Left: Coomassie blue stain; right: fluorescence illumination and densitometry of the fluorescence profiles. Untreated $(\cdots)$ (see lane 1); DTT treated ( $\longrightarrow$ ) (see lane 2).

of spermatozoa in man is short compared with that of other species (Amann and Howards, 1980). The duration of sperm transit in the human epididymis also varies among individuals (Rowley et al., 1970). The differences in sperm epididymal transit time may explain the differences among species in nuclear decondensation (Bedford et al., 1973) and heterogeneity in sperm thiols (Seligman et al., 1991). Staining of spermatozoa of human and other mammalian species with acridine orange has been shown to result in red or green (or intermediate) fluorescence of the nuclei; sperm nuclei of fertile males exhibit green acridine orange fluorescence, whereas sperm nuclei of infertile males exhibit red fluorescence after heat or acid denaturation, suggesting that chromatin structure influences the acridine orange-DNA interaction (Evenson et al., 1980; Tejada et al., 1984; Spano and Evenson, 1993). Acridine orange fluorescence has recently been shown to change with sperm maturation and to be determined by the thiol-disulfide status of sperm nuclei (Kosower et al., 1992). Immature sperm nuclei of several species, which contain mostly thiols, exhibit red acridine orange fluorescence; mature sperm nuclei, which contain mostly disulfides, exhibit green fluorescence. The fluorescence type can be altered by modulation of the sperm thiol-disulfide status (i.e., red acridine orange fluorescence upon disulfide reduction and green acridine orange fluorescence 


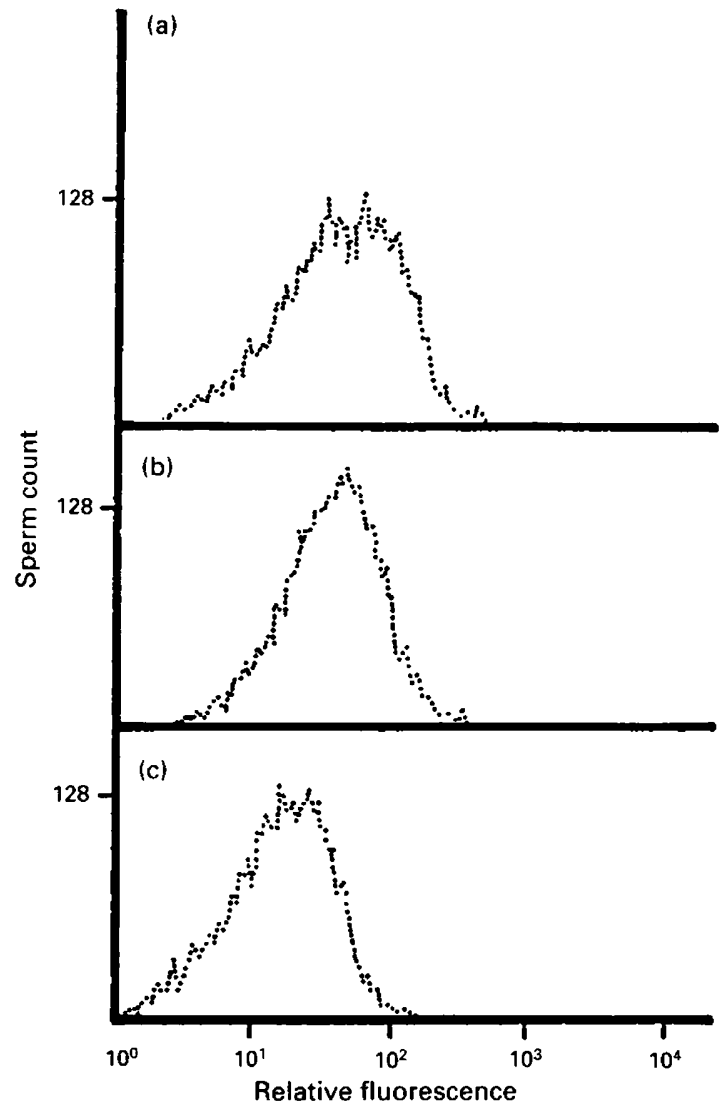

Fig. 7. Flow cytometry histograms of a human normozoospermic sample labelled with monobromobimane (mBBr). (a) Washed spermatozoa labelled immediately after washing; (b) Washed spermatozoa incubated at $4^{\circ} \mathrm{C}$ for 3 days in buffer containing $10 \mathrm{mmol}$ Tris- $\mathrm{HCl}$ $1^{-1}, 140 \mathrm{mmol} \mathrm{NaCl}^{-1}$, pH 7.4 (TBS) before labelling; (c) unwashed spermatozoa incubated in seminal fluid at $4^{\circ} \mathrm{C}$ for 3 days before labelling.

after thiol oxidation). Heterogeneity in acridine orange fluorescence is displayed by human spermatozoa and is also related to the sperm nuclei thiol-disulfide status (Kosower et al., 1992).

In the present work, normozoospermic samples demonstrated heterogeneity in thiol content among cells within the samples, as observed by microscopy and by flow cytometry histograms. The observed heterogeneity in sperm thiol content was not related to sperm morphology. The differences in sperm thiols shown in the present work indicate differences in the degree of sperm maturation among and within semen samples. The results are consistent with the reported differences in the extent of sperm nuclear condensation and in epididymal sperm transit time. It should be noted, however, that although the fertilizing capacity of spermatozoa recovered from human epididymal cauda is high compared with that of caput spermatozoa (Hinrichsen and Blaquier, 1980), the movement of spermatozoa through the epididymis may not be an absolute requirement for sperm maturation. Silber (1989) showed that caput spermatozoa, obtained from patients with obstructive azoospermia caused by blockage at the caput epididymis, can fertilize eggs. We found that in rats, spermatozoa accumulating proximally to ligated caput epididymis gain motility and fertilizing ability and that protein thiols in these spermatozoa are oxidized (Seligman $e t$ al., 1992). These studies indicate that spermatozoa may only require a period within certain segments of the epididymis to mature and gain fertilizing ability.

The chromatin of human spermatozoa consists of histones (15\%) and protamines (85\%) (Gatewood et al., 1987). The protamines include protamine $I$ (P1) and protamine 2 (P2), which differ in their molecular mass, amino acid composition and sequence (Gusse et al., 1986; McKay et al., 1986). PI is represented by only one molecular species (HP1). P2 includes several proteins (HP2, HP3 and HP4) (Gusse et al, 1986; Arkhis et al., 1991). Under the experimental conditions used here, HP1 and HP2 were identified. Electrophoretic analysis of protamines isolated from mBBr-labelled normozoospermic samples showed that the protamine bands contain some free reactive thiol groups and a high content of disulfides, as demonstrated by the significant increase in fluorescence of the protamines isolated from DTT-pretreated, mBBr-labelled spermatozoa. The finding of less thiol labelling in P2 than in P1 in the normozoospermic samples is in agreement with previous results on fewer cysteine residues and more histidine residues in P2 (Perreault et al., 1988). The presence of free thiols in the protamines and the differences in thiol content among protamines of the various samples is consistent with our observations by microscope and flow cytometry analyses of intact spermatozoa.

Abnormalities in chromatin structure may affect nuclear stabilization and decondensation and be associated with infertility (Evenson et al., 1980; Huret, 1986). Some recent studies have demonstrated alterations in HP1:HP2 and in nonprotamine:protamine ratios in some groups of infertile men (Balhorn et al., 1988; Bach et al., 1990). No information has been published on the protamine thiol-disulfide status of the infertile men. In the present study, we observed an increase in the ratio of non-protamine basic proteins:protamines in some teratozoospermic samples and a diminished HP1:HP2 ratio in some asthenozoospermic samples. The thiol content of the protamines isolated from teratozoospermic samples was in the same range as that of protamines in most normozoospermic samples, with similar variability among the samples. In contrast, protamines extracted from the asthenozoospermic samples contained no or very little free reactive thiols. The total SH plus SS in protamines of these samples was similar to that of the normozoospermic samples, indicating that the protamines of the asthenozoospermic samples contained mostly or only disulfides.

After penetration of the egg by the spermatozoa the time required for sperm nuclear decondensation and pronucleus formation depends in part on the sperm disulfide content (Perreault et al., 1988). Thus, spermatozoa with 'too high' content of disulfides would have a hyperstabilized chromatin, preventing the sperm nucleus from decondensing in the oocyte (Huret, 1986). Overstability of nuclear chromatin in ovine spermatozoa was found to be associated with diminished fertilizing ability (Rodrigues et al., 1985). Our finding of increased protamine oxidation in the asthenozoospermic samples is consistent with the proposed relation of protamine structure and thiol status to sperm fertilizing ability.

Little information is available on the human sperm tail proteins. It has been shown that rat and human tail accessory fibres consist of similar polypeptides and that human dense 
fibres contain protein subunits of $28 \mathrm{kDa}$ and $31 \mathrm{kDa}$ (Baccetti et al., 1976). In the study reported here, the protein bands shown by SDS-PAGE are non-basic proteins, as basic proteins do not enter this gel. The SDS-PAGE protein profile shows mainly tail and midpiece proteins (i.e. proteins present in sufficient concentrations to be detected as bands by the analytical procedures used here). In preliminary immunoblotting experiments, we found that anti-tubulin antibodies reacted with several protein bands in the range between $60 \mathrm{kDa}$ and $90 \mathrm{kDa}$ and with the $16 \mathrm{kDa}$ band (unpublished data). Further study is necessary for the identification of these proteins in human spermatozoa.

Thiols and disulfides have been demonstrated in the dense fibres of the sperm tail, connecting piece, fibrous sheath and outer mitochondrial membrane in various species (Bedford and Calvin 1974; Baccetti et al., 1976; Pallini et al., 1979), but no information is available on the thiol-disulfide status of human sperm tail proteins. The fluorescence profile of the SDS-PAGE of solubilized mBBr-labelled human spermatozoa showed that in the normozoospermic samples the proteins contain both thiols and disulfides and that the magnitude of thiol oxidation differs among proteins. The proteins of asthenozoospermic samples seem to be more oxidized, as they contain few free reactive thiols, but contain the same total of SH plus SS as those of the normozoospermic samples. It has been suggested that formation of disulfide bonds in dense fibres and fibrous sheath may play a role in the acquisition of sperm motility (Cornwell et al, 1988). The loss of sperm motility in asthenozoospermic samples may result from the 'overoxidation' of certain non-basic proteins.

The factors controlling thiol oxidation are yet to be identified. Epididymal components may play a role in sperm maturation and thiol oxidation (Tezon et al., 1985; Ross et al., 1990; Cooper, 1990). 'Hypercondensation' has been demonstrated in human spermatozoa aged in the epididymis (Calvin and Bedford, 1971). The 'overoxidation' and loss of motility of asthenozoospermic cells may arise from ageing in vivo or be caused by a defect in the epididymal environment. Another possibility is that the 'overoxidation' results from the exposure of spermatozoa to seminal plasma components. It is known that seminal plasma contains factors that inhibit sperm motility (Iwamoto and Gagnon, 1988). Similar seminal plasma factors may also lead to oxidation of thiols in the spermatozoa, as found in the study reported here. The observed effect of seminal plasma on the sperm thiol-disulfide status may be of importance for the preparation of spermatozoa for in vitro fertilization.

Semen quality is commonly evaluated by a number of routine parameters such as sample volume, cell count, morphology, motility and sometimes by additional means such as the ability to penetrate hamster eggs (Yanagimachi, 1988). Variations in fertilizing ability are not consistently correlated with normal semen parameters or with altered morphology or motility (Ballachey et al., 1987). Quantitative determination of solubilized sperm thiols showed variability among normozoospermic samples (Rufas et al., 1991). In this work we observed variability in the content of reactive thiols in the protamines among the normozoospermic group. Of particular interest is the sample that showed very high fluorescence, especially in the protamines. This sample was of a patient diagnosed as a case of unexplained infertility with normal semen parameters. This case may represent an example of sperm immaturity and infertility due to 'underoxidation'.

In conclusion, a correct degree of sperm thiol oxidation is thought to be important for sperm maturation, motility and activation and for the events during fertilization. This work demonstrates a simple method to follow thiols in intact spermatozoa and in different proteins in a single ejaculate. Our results suggest that an abnormal sperm thiol-disulfide status may be present in some cases of infertility.

This work was supported by grants from the Rockefeller Foundation and from the Chief Scientist, Israel Ministry of Health. The work formed part of a PhD thesis, Tel-Aviv University (J. Seligman).

\section{References}

Amann RP and Howards SS (1980) Daily spermatozoal production and epididymal spermatozoal reserves in the human male Joumal of Urology 124 211-215

Arkhis M, Martinage A, Sautiere P and Chevaillier P (1991) Molecular structure of human protamine P4 (HP4), a minor basic protein of human sperm nuclei European Journal of Biochemistry 200 387-392

Baccetti B, Pallini V and Burrini AG (1976) The accessory fibers of sperm tail III high-sulfur and low-sulfur components in mammals and cephalopods Joumal of Ultrastructure Research 57 289-308

Bach O, Glander HJ, Scholz GG and Schwarz J (1990) Electrophoretic patterns of spermatozoal nucleoproteins (NP) in fertile men and infertile patients and comparison with NP of somatic cells Andrologia 22 217-224

Balhorn R, Reed S and Tanphaichitr N (1988) Aberrant protamine 1/protamine 2 ratios in sperm of infertile human males Experientia 44 52-55

Ballachey BE, Hohenboken WD and Evenson DP (1987) Heterogeneity of sperm nuclear chromatin structure and its relationship to bull fertility Biology of Reproduction 36 915-925

Bedford JM and Calvin HI (1974) Changes in S-S linked structures of the sperm tail during epididymal maturation, with comparative observations in submammalian species Journal of Experimental Zoology 187 181-204

Bedford JM, Bent MJ and Calvin HI (1973) Variations in the structural character and stability of the nuclear chromatin in morphologically normal human spermatozoa Journal of Reproduction and Fertility 33 19-29

Calvin HI and Bedford JM (1971) Formation of disulphide bonds in the nucleus and accessory structures of mammalian spermatozoa during maturation in the epididymis Journal of Reproduction and Fertility Supplement 13 65-75

Cooper TG (1990) In defense of a function for the human epididymis Fertility and Sterility 54 965-975

Cornwall GA, Vindivich D, Tillman S and Chang TSK (1988) The effect of sulfhydryl oxidation on the morphology of immature hamster epididymal spermatozoa induced to acquire motility in vitro Biology of Reproduction 39 141-155

Evenson DP, Darzynkiewicz Z and Melamed MR (1980) Relation of mammalian sperm chromatin heterogeneity to fertility Science 210 1131-1133

Fleming AD, Kosower NS and Yanagimachi R (1986) Alteration of sperm thiol-disulfide status and capacitation in the guinea pig Gamete Research 13 93-102

Gatewood JM, Cook GR, Balhorn R, Bradbury EM and Schmid CW (1987) Sequence-specific packaging of DNA in human sperm chromatin Science 236 962-964

Gusse M, Sautiere P, Belaiche D, Martinage A, Roux C, Dadoune JP and Chevaillier $P$ (1986) Purification and characterization of nuclear basic proteins of human sperm Biochimica et Biophysica Acta 884 124-134

Hinrichsen MJ and Blaquier JA (1980) Evidence supporting the existence of sperm maturation in the human epididymis Joumal of Reproduction and Fertility 60 291-294

Huang TTF, Kosower NS and Yanagimachi R (1984) Localization of thiol and disulfide groups in guinea pig spermatozoa during maturation and capacitation using bimane fluorescent labels Biology of Reproduction 31 797-809

Huret JL (1986) Nuclear chromatin decondensation of human sperm: a review Archives of Andrology $1697-109$ 
Iwamoto $T$ and Gagnon C (1988) Purification and characterization of a sperm motility inhibitor in human seminal plasma Journal of Andrology 9 377-383

Kosower NS and Kosower EM (1987) Thiol labeling with bromobimanes Methods of Enzymology 143 76-84

Kosower NS, Katayose H and Yanagimachi R (1992) Thiol-disulfide status and acridine orange fluorescence of mammalian sperm nuclei Journal of Andrology $13342-348$

Laemmli UK (1970) Cleavage of structural proteins during the assembly of the head of bacteriophage T4 Nature 227 680-685

McKay DJ, Renaux BS and Dixon GH (1986) Human sperm protamines: amino-acid sequences of two forms of protamine P2 European Journal of Biochemistry 156 5-8

Marushige $Y$ and Marushige $K$ (1975) Transformation of sperm histones during formation and maturation of rat spermatozoa Joumal of Biological Chemistry $25039-45$

Miller M and Masui Y (1982) Changes in the stainability and sulfhydryl level in the sperm nucleus during sperm-oocyte interaction in mice Gamete Research 5 167-180.

Pallini V, Baccetti B and Burrini AG (1979) A peculiar cysteine rich polypeptide related to some unusual properties of mammalian sperm mitochondria. In The Spermatozoon pp 141-150 Eds DW Fawcett and JM Bedford. Urban and Schwarzenberg Inc, Baltimore-Munich

Panyim S and Chalkley R (1969) High resolution acrylamide gel electrophoresis of histones Archives of Biochemistry and Biophysics 130 337-346

Pellicciari C, Hosokawa Y, Fukuda M and Romanini MG (1983) Cytofluorimetric study of nuclear sulphydryl and disulphide groups during sperm maturation in the mouse Journal of Reproduction and Fertility 68 371-376

Perreault SD, Barbee RR, Elstein KH, Zucker RM and Keefer CL (1988) Interspecies differences in the stability of mammalian sperm nuclei assessed in vivo by sperm microinjection and in vitro by flow cytometry Biology of Reproduction $39 \quad 157-167$

Rodriguez H, Ohanian C and Bustos-Obregon E (1985) Nuclear chromatin decondensation of spermatozoa in vitro: a method for evaluating the fertilizing ability of ovine semen International Joumal of Andrology 8 147-158

Ross P, Kan FWK, Antaki P, Vigneault N, Chapdelaine A and Roberts KD (1990) Protein synthesis and secretion in human epididymis and immunoreactivity with sperm antibodies Molecular Reproduction and Development 26 12-23
Rowley M, Teshima JF and Heller CG (1970) Duration of transit of spermatozoa through the human male ductular system Fertility and Sterility 21 390-397

Rufas O, Fisch B, Seligman J, Tadir Y, Ovadia J and Shalgi R (1991) Thiol status in human sperm Molecular Reproduction and Development 29 282-288

Seligman J and Shalgi R (1991) Protein thiols in rat sperm and epididymal fluid Journal of Reproduction and Fertility 93 399-408

Seligman J, Shalgi R, Oschry Y and Kosower NS (1991) Sperm analysis by fluorescence activated cell sorter using the fluorescent thiol labeling agent monobromobimane Molecular Reproduction and Development 29 276-281

Seligman J, Kosower NS and Shalgi R (1992) Effect of caput ligation on rat sperm and epididymis: protein thiols and fertilizing ability Biology of Reproduction 46 301-308

Shalgi R, Seligman J and Kosower NS (1989) Dynamics of the thiol status of rat spermatozoa during maturation: analysis with the fluorescent labeling agent monobromobimane Biology of Reproduction 40 1037-1045

Silber SJ (1989) Apparent fertility of human spermatozoa from caput epididymis Journal of Andrology 10 263-269

Spano M and Evenson DP (1993) Flow cytometric analysis for reproductive biology Biology of the Cell 78 53-62

Tejada RI, Mitchell JC, Normal A, Marik JJ and Friedman S (1984) A test for the practical evaluation of male fertility by acridine orange (AO) fluorescence Fertility and Sterility 42 87-91

Tezon JG, Ramella E, Cameo MS, Vazquez MH and Blaquier JA (1985) Immunocytochemical localization of secretion antigens in human epididymis and their association with spermatozoa Biology of Reproduction 32 591-597

World Health Organization (1980) Laboratory Manual for the Examination of Human Semen-Cervical Mucus Interaction. Press Conam, Singapore

Yanagimachi R (1988) Mammalian fertilization. In The Physiology of Reproduction pp 135-185 Eds E Knobil, J Neill, LL Ewing, GS Greenwald, C Market and $W$ Paff Raven Press, New York

Yanagimachi R, Huang TTF, Fleming AD, Kosower NS and Nicolson GL (1983) Dithiothreitol, a disulfide-reducing agent, inhibits capacitation, acrosome reaction, and interaction with eggs by guinea pig spermatozoa Gamete Research 7 145-154 